\title{
P01-7-22 Poster session
}

\section{Nursing practice and clinical pharmacology of prednisolone therapy for children with nephrotic syndrome}

\author{
Shunsuke Kanayama ${ }^{1}$, Haruka Aoto ${ }^{2}$, Akiko Matsuda $^{3}$, Yusuke Okamoto ${ }^{4}$, Keiichi Hanaki $^{2}$ \\ ${ }^{1}$ Department of Nursing, The University of Shimane, Japan, ${ }^{2}$ School of Health Sciences, Tottori University, Yonago, \\ Japan, ${ }^{3}$ Nara Medical University, Kashihara, Japan, ${ }^{4}$ Tottori University Hospital, Yonago, Japan
}

\section{Background}

In $80 \%$ of patients with childhood-onset idiopathic nephrotic syndrome (INS), remission is achieved by treatment with prednisolone. However, serious adverse effects and medication management incidents have been reported for prednisolone. Problems with medication management might be related to delayed treatment and relapse of INS. Therefore, it is important for nurses managing INS to have sufficient knowledge of clinical pharmacology and to understand the treatment regimens for this disease.

Objective

This study was performed to investigate nursing practice and knowledge of clinical pharmacology by comparison between two groups of nurses with or without experience in caring for patients with INS.

Design/Methods

Six hundred and ninety-seven nurses working at public hospitals and regional support hospitals with over four hundred beds were asked to enroll in this study. The questionnaire was developed with reference to the treatment guidelines for childhood-onset INS and the package insert for prednisolone (warnings, contraindications, relative contraindications, drug interactions, adverse effects, pharmacokinetics, pharmacology, etc.).

Results

We analyzed the responses of 210 out of 228 nurses who consented to the study. In the group with experience of providing nursing care for patients with INS, $85.7 \%$ understood the INS treatment regimens, while $83.6 \%$ understood the treatment regimens in the group with no experience. There was no significant difference between the two groups. There were also no significant differences for the following: nurses who provided nursing care according to the plan (experience: 90.8\% vs. no experience: $89.1 \%$ ), nurses who checked the package insert of prednisolone (experience: $63.3 \%$ vs. no experience: $54.5 \%$ ), nurses who did not know all the relative contraindications for prednisolone (experience: $25.5 \%$ vs. no experience $20.0 \%$ ), and nurses who did not know all of the precautions for coadministration (experience: $30.6 \%$ vs. no experience $35.5 \%)$.

Conclusion

This study demonstrated that nurses caring for patients with childhood-onset INS do not have enough knowledge of clinical pharmacology, suggesting the need for more nursing education about the clinical pharmacology of INS. 